\title{
Effect of meal composition and cooking duration on the fate of sulforaphane following consumption of broccoli by healthy human subjects
}

\author{
Vanessa Rungapamestry ${ }^{1 *}$, Alan J. Duncan ${ }^{2}$, Zoë Fuller ${ }^{2}$ and Brian Ratcliffe ${ }^{1}$ \\ ${ }^{1}$ School of Life Sciences, The Robert Gordon University, St Andrew Street, Aberdeen AB25 $1 H G, U K$ \\ ${ }^{2}$ The Macaulay Institute, Craigiebuckler, Aberdeen, AB15 8QH, UK
}

(Received 26 June 2006 - Revised 18 October 2006 - Accepted 20 October 2006)

\begin{abstract}
The isothiocyanate, sulforaphane, has been implicated in the cancer-protective effects of brassica vegetables. When broccoli is consumed, sulforaphane is released from hydrolysis of glucoraphanin by plant myrosinase and/or colonic microbiota. The influence of meal composition and broccoli-cooking duration on isothiocyanate uptake was investigated in a designed experiment. Volunteers $(n 12)$ were each offered a meal, with or without beef, together with $150 \mathrm{~g}$ lightly cooked broccoli (microwaved $2.0 \mathrm{~min}$ ) or fully cooked broccoli (microwaved $5.5 \mathrm{~min}$ ), or a broccoli seed extract. They received $3 \mathrm{~g}$ mustard containing pre-formed allyl isothiocyanate (AITC) with each meal. Urinary output of allyl (AMA) and sulforaphane (SFMA) mercapturic acids, the biomarkers of production of AITC and sulforaphane respectively, were measured for $24 \mathrm{~h}$ after meal consumption. The estimated yield of sulforaphane in vivo was about 3-fold higher after consumption of lightly cooked broccoli than fully cooked broccoli. Absorption of AITC from mustard was about 1.3-fold higher following consumption of the meat-containing meal compared with the non meat-containing alternative. The meal matrix did not significantly influence the hydrolysis of glucoraphanin and its excretion as SFMA from broccoli. Isothiocyanates may interact with the meal matrix to a greater extent if they are ingested pre-formed rather than after their production from hydrolysis of glucosinolates in vivo. The main influence on the production of isothiocyanates in vivo is the way in which brassica vegetables are cooked, rather than the effect of the meal matrix.
\end{abstract}

Allyl isothiocyanate: Sulforaphane: Mercapturic acid: Meal composition

Epidemiological studies suggest that the cancer-protective effects of brassica vegetables may be partly related to their content of glucosinolates (van Poppel et al. 1999). During the preparation and cooking of brassica, followed by their ingestion and digestion in the upper alimentary tract, glucosinolates are hydrolysed to thiohydroxamate- $O$-sulfonate by a plant thioglucosidase, myrosinase (EC 3.2.1.147), which is released from ruptured plant cells. Thiohydroxamate- $O$-sulfonate is then immediately converted to different classes of metabolites depending on the properties of the hydrolysis medium (Fenwick \& Heaney, 1983). A further stage of hydrolysis of glucosinolates occurs in the colon under the action of colonic microbiota (Elfoul et al. 2001; Krul et al. 2002). Isothiocyanates, produced from hydrolysis of glucosinolates at a neutral $\mathrm{pH}$, are one of the main metabolites implicated in the cancer-protective effects of brassica (Zhang et al. 2006).

An understanding of the digestive and absorptive fate of dietary glucosinolates and their metabolites has emerged mostly from mechanistic studies in animal models (Brüsewitz et al. 1977; Mennicke et al. 1983; Duncan et al. 1997; Elfoul et al. 2001). A few studies, involving human subjects, have used urinary biomarkers to demonstrate the absorption of isothiocyanates in vivo after the intake of glucosinolates from brassica vegetables (Getahun \& Chung, 1999; Conaway et al. 2000; Shapiro et al. 2001; Rouzaud et al. 2004). After their absorption, isothiocyanates are metabolised by the mercapturic acid pathway in vivo. Isothiocyanates initially form conjugates with glutathione, then undergo enzymatic modifications, and are excreted in urine as their corresponding $\mathrm{N}$-acetylcysteine conjugates or mercapturic acids (MA) (Brüsewitz et al. 1977). Urinary excretion of MA of isothiocyanates therefore reflects their production and uptake in vivo.

The production of isothiocyanates following the ingestion of brassica is influenced by residual glucosinolate concentration and plant myrosinase activity in the ingested vegetable. However, brassica vegetables are normally consumed as part of a meal and the digestive fate of glucosinolates may be further influenced by the composition of the meal matrix.

Sulforaphane, the alkyl isothiocyanate in broccoli, is produced from hydrolysis of its alkyl glucosinolate precursor, glucoraphanin. The aim of the present study was to determine the effect of the meal matrix on the production of sulforaphane from lightly cooked or fully cooked broccoli in healthy human volunteers.

\section{Materials and methods}

\section{Human subjects}

Twelve healthy, non-smoking, human volunteers ( 5 males and 7 females), with mean BMI 26.1 (SEM 1.52$) \mathrm{kg} / \mathrm{m}^{2}$ and mean 
age 38.4 (SEM 3.11) years, were recruited for the study after giving their informed consent. None of the subjects was on any drug or dietary supplement just before the start of or during the experiment. Volunteers were low to moderate consumers of brassica vegetables. They were given information on the study and provided with a urine collection kit and instructions for the collection and storage of urine following their experimental meals. Volunteers were asked to avoid foods containing glucosinolates and isothiocyanates, and nutritional supplements for $48 \mathrm{~h}$ before their first experimental meal and throughout the study. They were also required to keep a simple food diary of their daily dietary intake during that period. Ethical approval for the study was obtained from the Grampian Research Ethics Committee within the Grampian Hospitals NHS Trust.

\section{Experimental design}

The experiment consisted of a $2 \times 3$ factorial design with factors involving a meal containing meat or a vegetarian equivalent, and three broccoli treatments comprising $150 \mathrm{~g}$ lightly cooked broccoli, $150 \mathrm{~g}$ fully cooked broccoli or an extract of broccoli seeds $(70 \mathrm{ml})$. Amounts of cooked broccoli corresponded to a portion size slightly larger than generally consumed (MAFF, 1988), while the amount of seed extract was adapted from Rouzaud (2001) and aimed to contain substantial concentrations of sulforaphane. These amounts were chosen to enhance the estimation of recovery of MA in urine. The vegetarian meal consisted of rice and a tomato and basil sauce, while the meat-containing alternative also contained lean, minced beef. The broccoli seed extract was a source of pre-formed metabolites of glucosinolates, generated from hydrolysis of glucosinolates during its preparation. It was designed to determine the recovery of metabolites of glucosinolates after their ingestion and absorption in vivo. Six experimental meals were offered to each volunteer. Meals were served on Mondays and Thursdays over 3 weeks, and were thus separated by a wash-out period of at least $48 \mathrm{~h}$ to avoid potential carry-over effects. Meals were allocated to volunteers by randomising type of meal (vegetarian or meatcontaining meal) and broccoli treatment (lightly cooked, fully cooked and seed extract) within two combined $6 \times 6$ complete Latin squares. The order of allocation of each combination of type of meal and cooking treatment of broccoli was balanced to resolve possible carry-over effects of dietary treatment. Since each individual received all combinations of type of meal and broccoli-cooking treatment, each subject was treated as a block in ANOVA to account for inter-individual variation in urinary excretion of MA.

Volunteers were offered $3 \mathrm{~g}$ English mustard with each experimental meal. Mustard is an abundant and stable source of preformed allyl isothiocyanate (AITC; Rouzaud et al. 2004). It was used to control for intra- and inter-individual variation in the absorption and excretion of isothiocyanates. Fig. 1 illustrates

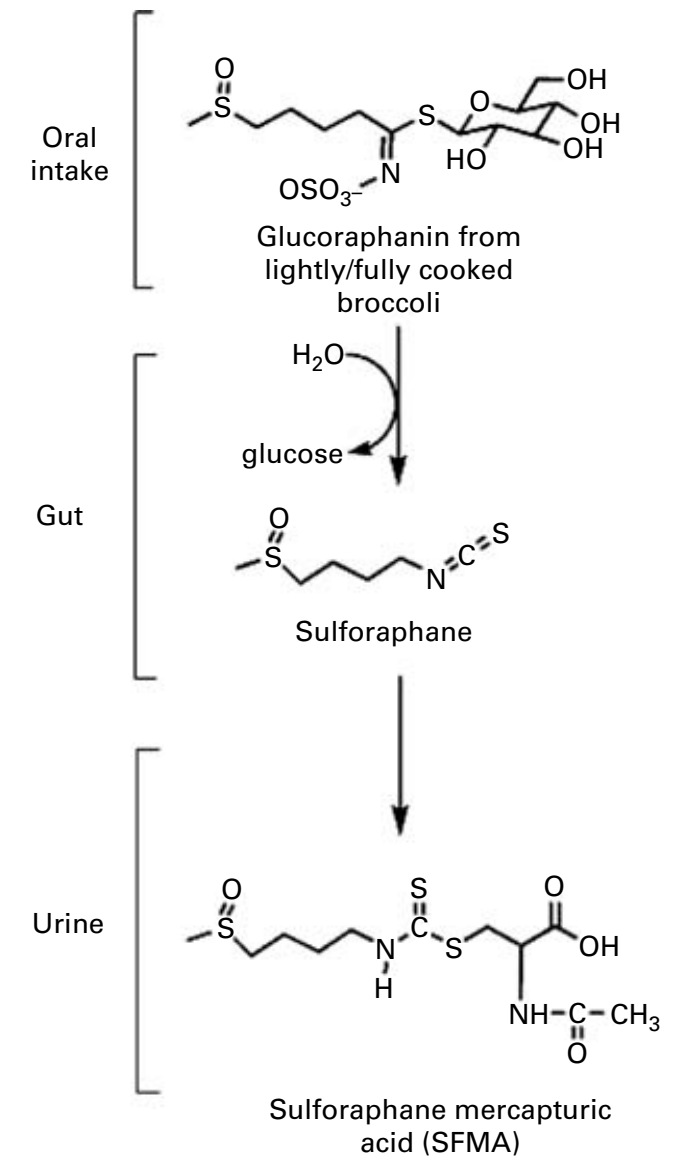

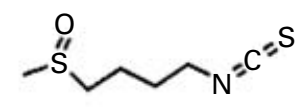

Sulforaphane from broccoli seed extract

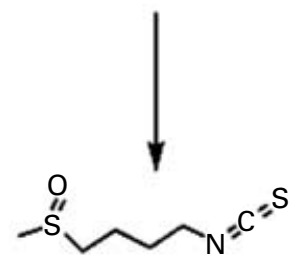

Sulforaphane

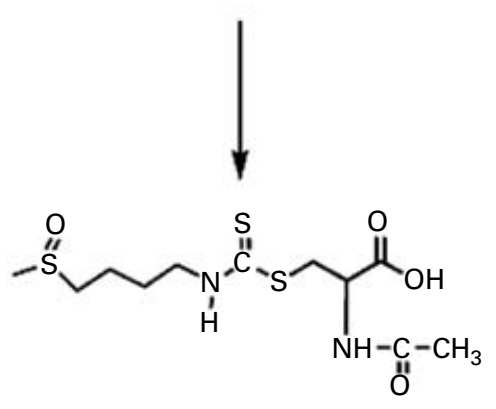

Sulforaphane mercapturic acid (SFMA)

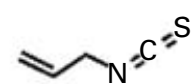

Allyl isothiocyanate (AITC) from mustard

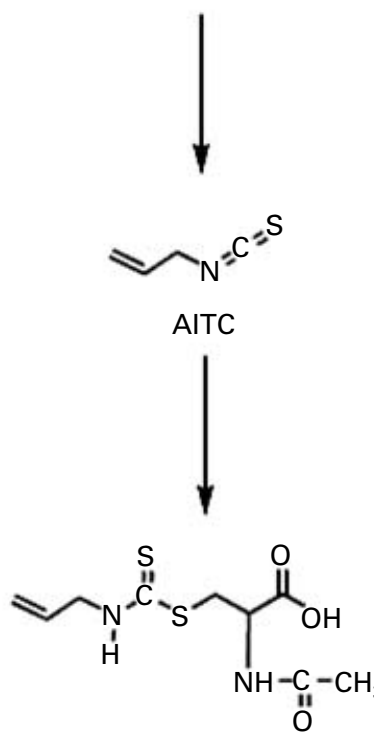

Allyl mercapturic acid (AMA)

Fig. 1. Metabolic fate of glucosinolate precursors and isothiocyanates following consumption of brassica within experimental meals by volunteers. 
the expected metabolic fate of glucosinolate precursors and isothiocyanates following consumption of brassica within the experimental meals by human volunteers.

\section{Experimental meals}

Broccoli heads (Var. Monaco), each weighing about $300 \mathrm{~g}$ and pre-packed with PVC film, were purchased from a local supermarket (Sainsbury's, Aberdeen, UK) on the evening before each experimental day and held at $4^{\circ} \mathrm{C}$ until they were cooked. The variety of broccoli and its wholesale source were kept constant throughout the experimental period. Broccoli florets were cut and maintained at $4{ }^{\circ} \mathrm{C}, 30 \mathrm{~min}$ before being cooked and served to volunteers. Florets were divided into portions, weighing $150 \mathrm{~g}$ each, from seven broccoli heads for each group of six individuals to obtain a uniform product within groups. Lightly cooked or fully cooked broccoli was prepared by cooking a portion of florets with $16 \mathrm{ml}$ tap water in a heat-resistant dish, covered with pierced PVC film, in a microwave oven at $750 \mathrm{~W}$ for 2.0 or $5.5 \mathrm{~min}$ respectively. Test samples of lightly cooked or fully cooked broccoli were prepared at the same time as broccoli that was offered to volunteers within each group. They were maintained at room temperature while volunteers consumed their meals, and frozen in liquid $\mathrm{N}_{2}$ to be stored at $-80^{\circ} \mathrm{C}$ for subsequent analysis of residual glucoraphanin concentration and plant myrosinase activity.

Forty portions of broccoli seed extract were prepared $2 \mathrm{~d}$ before the start of the experiment and were frozen at $-20^{\circ} \mathrm{C}$ for use throughout the experiment. GEO organic broccoli seeds (UK Juicers Ltd, York, UK) were milled in a coffee grinder. For each extract, $9 \mathrm{~g}$ ground broccoli seed was mixed with $50 \mathrm{ml}$ tap water and incubated for $1 \mathrm{~h}$ at room temperature to allow hydrolysis of glucoraphanin to sulforaphane and sulforaphane nitrile. The suspension was sieved and decanted into a polypropylene bottle. All bottles containing the extract were sealed and placed in a water bath at $80^{\circ} \mathrm{C}$ for 10 min to inactivate plant myrosinase (Rouzaud et al. 2004). Extracts were cooled on ice for $15 \mathrm{~min}$, followed by the addition of $20 \mathrm{ml}$ pure orange juice (Del Monte Foods International, Staines, Middx, UK) and $10 \mathrm{~g}$ Canderel (aspartame) artificial sweetener (Merisant UK Ltd, Bucks, UK) to improve palatability. The seed extracts were stored at $-20^{\circ} \mathrm{C}$ and defrosted at $4^{\circ} \mathrm{C}$ on the evening before each experimental meal.

The meat-containing and vegetarian meals were prepared and blast-frozen 2 weeks before the start of the experiment. For the meat-containing component, $5000 \mathrm{~g}$ fresh, lean minced beef topside was purchased from a local supermarket (Tesco, Aberdeen, UK). It was cooked in two equal batches, each in $10 \mathrm{ml}$ olive oil followed by the addition of $3000 \mathrm{~g}$ tomato and basil sauce (Bertolli UK, West Sussex, UK) and further cooking for $50 \mathrm{~min}$. For the vegetarian component, two batches of $3000 \mathrm{~g}$ tomato and basil sauce were each heated for $30 \mathrm{~min}$. Following cooking, the meat-containing and vegetarian components were portioned into $175 \mathrm{~g}$ and $100 \mathrm{~g}$ respectively, each type of meal thus containing equal amounts of sauce, and were blast frozen in heat-proof, disposable containers. They were stored at $-20^{\circ} \mathrm{C}$ and heated in an oven at $200^{\circ} \mathrm{C}$ for $20 \mathrm{~min}$ before being served to volunteers.
Basmati rice was cooked and held in a rice cooker $30 \mathrm{~min}$ before the experimental meals were served.

Portions of smooth English mustard (Colman's, Norwich, Norfolk, UK), weighing $30 \mathrm{~g}$ each, were incubated in a water bath at $80^{\circ} \mathrm{C}$ for $10 \mathrm{~min}$ to inactivate plant myrosinase. They were cooled on ice for $40 \mathrm{~min}$ and frozen at $-20^{\circ} \mathrm{C}$. Mustard was defrosted at $4^{\circ} \mathrm{C}$ on the evening before each experimental meal and volunteers received $3 \mathrm{~g}$ (actual amount, 3.011 (SEM 0.002) g at each meal.

\section{Urine collection}

Volunteers were asked to collect the total volume of one micturition just before consuming each experimental meal and then all urine excreted for $24 \mathrm{~h}$ after the meals. The latter was split into four batches, from $0-4 \mathrm{~h}, 4-10 \mathrm{~h}, 10-19 \mathrm{~h}$ and 19-24h after the experimental meals. Each batch was collected into separate containers which were sealed and kept in cool bags containing cool blocks. Total volume of urine excreted within each batch was measured on receipt of samples from volunteers and $20 \mathrm{ml}$-aliquots of each sample were stored at $-20^{\circ} \mathrm{C}$ for analysis of MA.

\section{Analysis of test meals}

Glucoraphanin in broccoli. Glucoraphanin was extracted from freeze-dried broccoli, desulfated and analysed by HPLC, as modified from Minchinton et al. (1982) and European Union (1997), and described by Rungapamestry et al. (2006). Glucoraphanin concentration was quantified using published response factors that have been experimentally determined (European Union, 1997). A standard curve was also constructed for glucoraphanin (KVL, Frederiksberg, Denmark) at concentrations from 0 to $20 \mu \mathrm{mol} / \mathrm{g} \mathrm{DM}$ within a freeze-dried watercress matrix $\left(r^{2} 0.9905\right)$ to verify linearity over the analytical range. The limit of quantification for desulfoglucoraphanin was $0.044 \mu \mathrm{mol} / \mathrm{g}$ DM.

Metabolites of glucosinolates in mustard and broccoli seed extract. AITC from mustard, and sulforaphane and sulforaphane nitrile from broccoli seed extract were analysed using the methods of Rouzaud et al. (2004), modified as follows. AITC was extracted twice in $10 \mathrm{ml}$ dichloromethane from $1 \mathrm{~g}$ mustard diluted in $10 \mathrm{ml}$ deionised water, which contained $100 \mu \mathrm{l} 67 \mathrm{~mm}$ butyl isothiocyanate (Aldrich Chemical Company, Milwaukee, WI, USA) as internal standard. Sulforaphane and sulforaphane nitrile were extracted from $5 \mathrm{ml}$ broccoli seed extract, which contained $100 \mu \mathrm{l} 250 \mathrm{~mm}$ benzyl isothiocyanate (Aldrich Chemical Company) as internal standard. In each case, the pooled dichloromethane extracts were concentrated to $100 \mu \mathrm{l}$. Metabolites of glucosinolates were analysed by GC with a flame ionisation detector, using an Equity-1 fused silica capillary column with a non-polar poly(dimethylsiloxane) bonded phase $30 \mathrm{~m} \times 0.25 \mathrm{~mm}$ i.d. $\times 0.25 \mu \mathrm{m}$ film thickness (Supelco, Bellefonte, PA, USA). AITC was analysed at an isothermal column temperature of $70^{\circ} \mathrm{C}$ for $20 \mathrm{~min}$, as adapted from Rouzaud et al. (2004). He gas was used as a carrier at a constant flow rate of $1 \mathrm{ml} / \mathrm{min}$ and a split flow rate of $30 \mathrm{ml} / \mathrm{min}$. The injector and detector were held at $200^{\circ} \mathrm{C}$. For sulforaphane and sulforaphane nitrile, the initial temperature of the column was maintained at $150^{\circ} \mathrm{C}$ for $2 \mathrm{~min}$, and then increased linearly at 
$10^{\circ} \mathrm{C} / \mathrm{min}$ to $270^{\circ} \mathrm{C}$, and was maintained for $10 \mathrm{~min}$ at $270^{\circ} \mathrm{C}$ (Matusheski et al. 2004).

An authentic standard of AITC (Sigma-Aldrich, St Louis, MO, USA) was extracted at nominal concentrations from 3 to $30 \mu \mathrm{mol} / \mathrm{ml}$ within an aqueous matrix ( $r^{2}$ value for calibration curve was 0.9999). Sulforaphane and sulforaphane nitrile (synthesised by N. P. Botting, Department of Chemistry, University of St Andrews, UK) were added, at nominal concentrations from 0 to 0.68 and 0 to $3.14 \mu \mathrm{mol} / \mathrm{ml}$ respectively, to an extract of ground mustard seed, prepared using the same protocol as for the broccoli seed extract. They were then extracted from the mustard seed extract to construct a standard curve within a matrix similar to the broccoli seed extract ( $r^{2}$ values for calibration curves were 0.9961 and 0.9971 respectively). Metabolites of glucosinolates had an extraction efficiency of about $98 \%$ from a freeze-dried vegetable matrix and their concentrations were not corrected for this efficiency since the internal standard was recovered to a similar extent.

Myrosinase activity. The determination of myrosinase activity in broccoli was adapted from Shapiro et al. (2001), as described by Rungapamestry et al. (2006). A standard curve was prepared using myrosinase from a commercial source (Sigma-Aldrich) ( $r^{2}$ 0.9930). Myrosinase activity was expressed as units/portion of cooked broccoli (1 unit myrosinase will produce $1 \mu \mathrm{mol}$ glucose from hydrolysis of $1 \mu \mathrm{mol}$ 2-propenyl glucosinolate at $25^{\circ} \mathrm{C}$ and $\mathrm{pH}$ 6.0).

Macronutrient analysis in meat-containing and vegetarian meals. $\mathrm{N}$ content in freeze-dried test meals of the mince and tomato and basil sauce component of the meat-containing meal and the tomato and basil sauce component of the vegetarian meal was determined by the Dumas Combustion method (Pella \& Colombo, 1973). Crude protein content was calculated by multiplying the $\mathrm{N}$ content by a factor of 6.25. Total fat content in freeze-dried test meals was determined by gravimetric extraction with iso-hexane for $6 \mathrm{~h}$ (British Standards, 1996).

Analysis of urinary mercapturic acids. $\quad N$-acetyl- $S$ - $(N-4-$ methylsulfinylbutylthiocarbamoyl)-L-cysteine (SFMA) and $N$-Acetyl- $S$-( $N$-allylthiocarbamoyl)-L-cysteine (AMA), the mercapturic acids of sulforaphane and AITC respectively, were extracted from human urine and analysed by HPLC according to Rouzaud et al. (2004), as adapted from analysis of MA in rat urine (Mennicke et al. 1987). The dicyclohexylamine salt of $N$-Acetyl-S-(N-propylthiocarbamoyl)-L-cysteine or propyl mercapturic acid (PMA) (synthesised according to Mennicke et al. (1983)) was used as the internal standard. Urinary SFMA and AMA concentrations were determined from calibration curves of SFMA (synthesised by N. P. Botting, Department of Chemistry, University of St Andrews, UK) and the dicyclohexylamine salt of AMA (synthesised according to Mennicke et al. (1983)) ( $r^{2}$ values of 0.9976 to 1.000 for AMA and 0.9865 to 1.000 for SFMA). The purity of SFMA standard was unknown and absolute concentrations of SFMA were calculated, assuming SFMA had a similar response to AMA and PMA in the UV detector (Rouzaud, 2001).

\section{Calculations and statistical analysis}

Samples were analysed in duplicate and data were presented as means with their standard errors for a minimum of six observations for analysis of test meals and twelve observations for MA analysis. Statistical analyses were performed using the software GenStat Release 8.1 (Genstat 5 Committee, 2005). The data for excretion of AMA and SFMA were analysed and interpreted using two approaches. Firstly, total molar output of AMA or SFMA, for $24 \mathrm{~h}$ following consumption of meals, was expressed as a proportion of the molar intake of their ingested precursors. AMA output was calculated as a proportion of AITC intake and, SFMA output, as a proportion of glucoraphanin or sulforaphane intake. The effect of broccoli-cooking treatment and meal composition on total AMA or SFMA output as a proportion of ingested precursors was tested by ANOVA. Since each volunteer received all combinations of the dietary treatments, the significance of cooking treatment and meal matrix was assessed within volunteers. The inter-individual variability in MA excretion as a proportion of precursor ingested was tested by ANOVA.

Secondly, the amount of AMA or SFMA excreted in urine collected during each of the four periods, following consumption of the meals, was expressed as a proportion of the amount of precursors consumed. The effect of dietary treatment and time of urine collection on MA excretion after meal consumption was tested by ANOVA. The difference in mean MA output between the four periods of their excretion after meal consumption was tested using orthogonal contrasts within ANOVA at $P<0 \cdot 001$.

\section{Results \\ Composition of experimental meals}

Intake of glucosinolates, isothiocyanates and myrosinase. Glucoraphanin concentration comprised approximately $20 \%$ $(\mu \mathrm{mol} / \mu \mathrm{mol})$ total glucosinolate concentration in cooked broccoli. Mean glucoraphanin intake by volunteers was not significantly different between lightly cooked broccoli (62.0 (SEM 6.17) $\mu \mathrm{mol} /$ portion) and fully cooked broccoli $(71.7$ (SEM 5.73) $\mu \mathrm{mol} /$ portion) $(P=0.301)$. The extract of broccoli seeds contained 2.7 (SEM 0.63) $\mu \mathrm{mol}$ sulforaphane and 121.6 (SEM 4.72) $\mu$ mol sulforaphane nitrile/portion, while mustard contained 51.9 (SEM 0.08) $\mu \mathrm{mol}$ AITC/portion. Intake of plant myrosinase by volunteers was significantly higher after lightly cooked broccoli (55.7 (SEM 4.69) units/portion) than fully cooked broccoli (15.6 (SEM 1.17) units/portion) $(P<0.001)$.

Macronutrient composition of meat-containing and vegetarian meals. The two types of meals were significantly different in their crude protein $(P<0.001)$ and total fat content $(P<0.001)$. The meat-containing and vegetarian components of the experimental meals contained $26 \cdot 7$ (SEM 0.41) and $2 \cdot 2$ (SEM 0.01) g protein/portion respectively. Total fat content was 7.4 (SEM 0.03) and 1.5 (SEM 0.02) g/portion respectively.

\section{Excretion of mercapturic acids}

Kinetics of allyl mercapturic acid and sulforaphane mercapturic acid output. The kinetics of AMA and SFMA excretion in urine collected following the experimental meals are shown in Fig. 2 and Fig. 3 respectively. Excretion of AMA and SFMA by volunteers was complete $24 \mathrm{~h}$ after consumption 

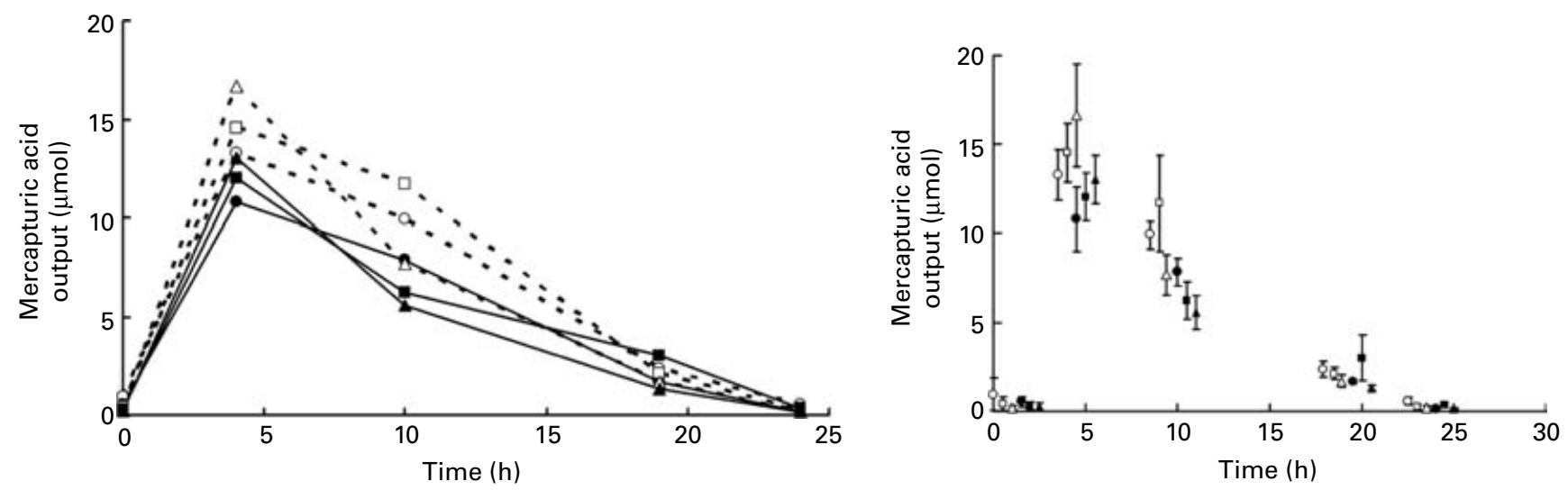

Fig. 2. Excretion of allyl mercapturic acid (AMA; $\mu \mathrm{mol})$, in baseline urine at time 0 before the experimental meals and from $0-4,4-10,10-19$ and $19-24 \mathrm{~h}$ after each meal. Experimental meals consisted of lightly cooked broccoli with a meat-containing $(O)$ or vegetarian $(\bullet)$ meal, fully cooked broccoli with a meat-containing $(\square)$ or vegetarian $(\square)$ meal, and broccoli seed extract with a meat-containing $(\Delta)$ or vegetarian $(\mathbf{\Lambda})$ meal. Each point represents the mean of twelve observations. Mean values with their standard errors depicted by vertical bars are presented separately for clarity.
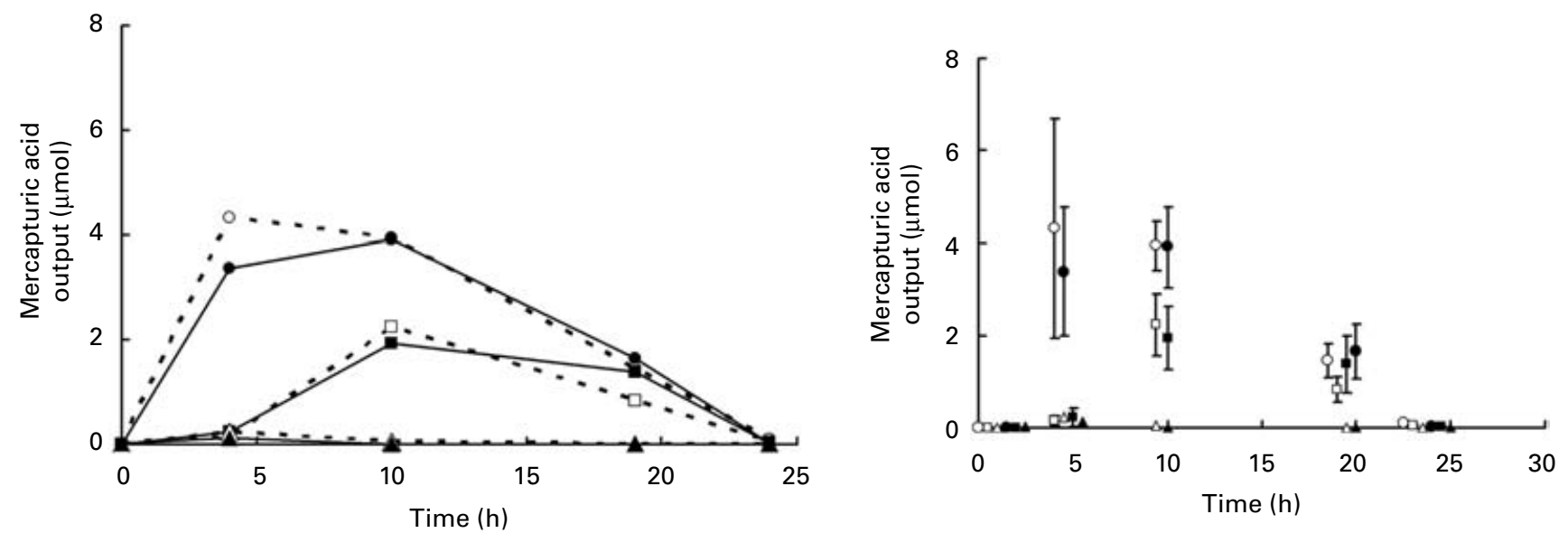

Fig. 3. Excretion of sulforaphane mercapturic acid (SFMA; $\mu \mathrm{mol})$, in baseline urine at time 0 before the experimental meals and from 0-4, 4-10, 10-19 and 19$24 \mathrm{~h}$ after each meal. Experimental meals consisted of lightly cooked broccoli with a meat-containing $(O)$ or vegetarian $(\bullet)$ meal, fully cooked broccoli with a meat-containing $(\square)$ or vegetarian $(\square)$ meal, and broccoli seed extract with a meat-containing $(\triangle)$ or vegetarian $(\boldsymbol{\Delta})$ meal. Each point represents the mean of twelve observations. Mean values with their standard errors depicted by vertical bars are presented separately for clarity.

of the experimental meals. Trace concentrations of SFMA were observed after consumption of the broccoli seed extract.

Total allyl mercapturic acid and sulforaphane mercapturic acid output as a proportion of ingested precursors. Total AMA output over $24 \mathrm{~h}$, as a proportion of AITC intake, was significantly higher after consumption of the meat-containing meal compared with the vegetarian meal by volunteers $(P<0.001)$, but was not influenced by broccoli treatment $(P=0.563)$ (Fig. 4(a)). Total SFMA output over $24 \mathrm{~h}$, as a proportion of glucoraphanin intake, was significantly higher after consumption of lightly cooked than fully cooked broccoli by volunteers $(P<0.001)$ (Fig. 4(b)). SFMA output was not affected by meal composition $(P=0.459)$. The average proportion of AITC in mustard absorbed and excreted as AMA (Fig. 4(a)) was higher than the mean fraction of glucoraphanin hydrolysed and excreted as SFMA after the consumption of cooked broccoli (Fig. 4(b)). Both total AMA and SFMA output, expressed as a proportion of precursor ingested, varied significantly among volunteers $(P<0 \cdot 001)$. As determined from the $\mathrm{CV}$ among individuals, SFMA output was about 2- and 4-fold more variable than AMA output after consumption of lightly cooked broccoli with the meat-containing and vegetarian meals respectively $(P<0 \cdot 001)$. After consumption of fully cooked broccoli with the meat-containing or vegetarian meals, SFMA output was about 3- or 4-fold more variable than AMA output $(P<0 \cdot 001)$ (Fig. 5a,b).

Rate of allyl mercapturic acid and sulforaphane mercapturic acid output as a proportion of intake of precursor. Approximately $50 \%$ AITC ingested was recovered as AMA $24 \mathrm{~h}$ after consumption of the meat-containing meal compared with about $40 \%$ after the vegetarian meal $(P=0.001)$. AMA output was highest $4 \mathrm{~h}$ after consumption of the meals $(P<0.001)$, with about one-third of AITC ingested being excreted in that time. The proportion of AITC absorbed and excreted as AMA after dietary intake was then significantly reduced over time $(P<0.001)$. The proportion of AITC excreted was 2-fold lower in the second than in the first urine collection when mustard was ingested along with fully cooked broccoli and the vegetarian meal $(P<0 \cdot 001)$, but was similar in the first two urine collections after its consumption with fully cooked 

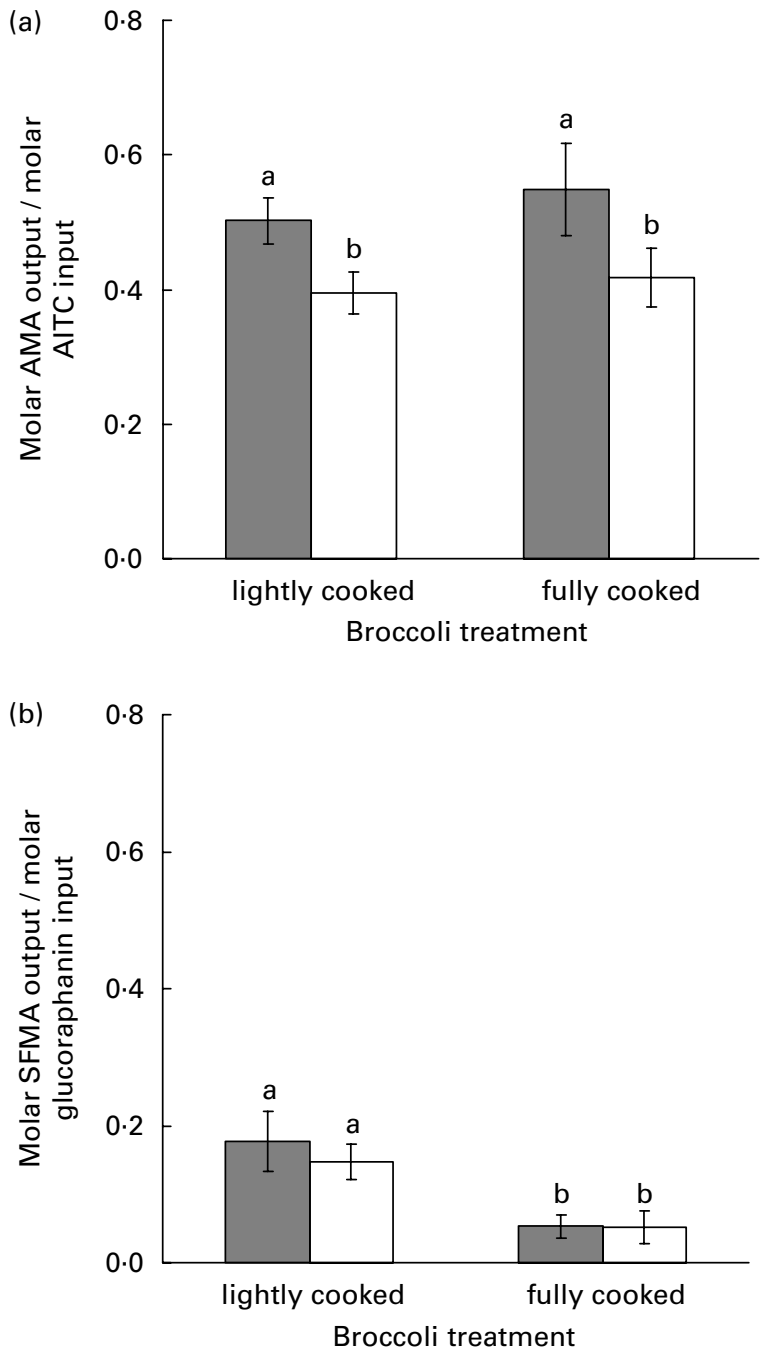

Fig. 4. Total excretion of (a) allyl mercapturic acid (AMA; $\mu \mathrm{mol}$ ) over $24 \mathrm{~h}$ as a proportion of allyl isothiocyanate (AITC) intake $(\mu \mathrm{mol})$, and (b) sulforaphane mercapturic acid (SFMA; $\mu \mathrm{mol}$ ) over $24 \mathrm{~h}$ as a proportion of glucoraphanin intake $(\mu \mathrm{mol})$, after consumption of lightly cooked or fully cooked broccoli along with a meat-containing $(\square)$ or vegetarian $(\square)$ meal. Values are means for twelve replicates, with their standard errors represented by vertical bars. ${ }^{\mathrm{a}, \mathrm{b}}$ Mean values with different letters were significantly different at $P<0.001$. Allyl mercapturic acid (AMA) output was significantly affected by type of meal $(P<0.001)$. Sulforaphane mercapturic acid (SFMA; $\mu \mathrm{mol})$ output was significantly influenced by broccoli treatment $(P<0.001)$.

broccoli and the meat-containing meal $(P=0 \cdot 181)$. This led to an interaction between the effect of type of meal and time of urinary excretion on AMA output $(P=0 \cdot 020)$.

The proportion of glucoraphanin hydrolysed to sulforaphane and excreted as SFMA $24 \mathrm{~h}$ after the meals was approximately $20 \%$ from lightly cooked broccoli and $5 \%$ from fully cooked broccoli. SFMA was not recovered after consumption of the broccoli seed extract. Excretion of SFMA, as a proportion of glucoraphanin intake, was highest $4-10 \mathrm{~h}$ after intake of lightly cooked or fully cooked broccoli and was subsequently reduced over time $(P<0.001)$. Most of the hydrolysed glucoraphanin was excreted as SFMA in the first and second urine collections following consumption of lightly cooked broccoli but in the second and third urine collections after intake of fully cooked broccoli. After the intake of lightly cooked
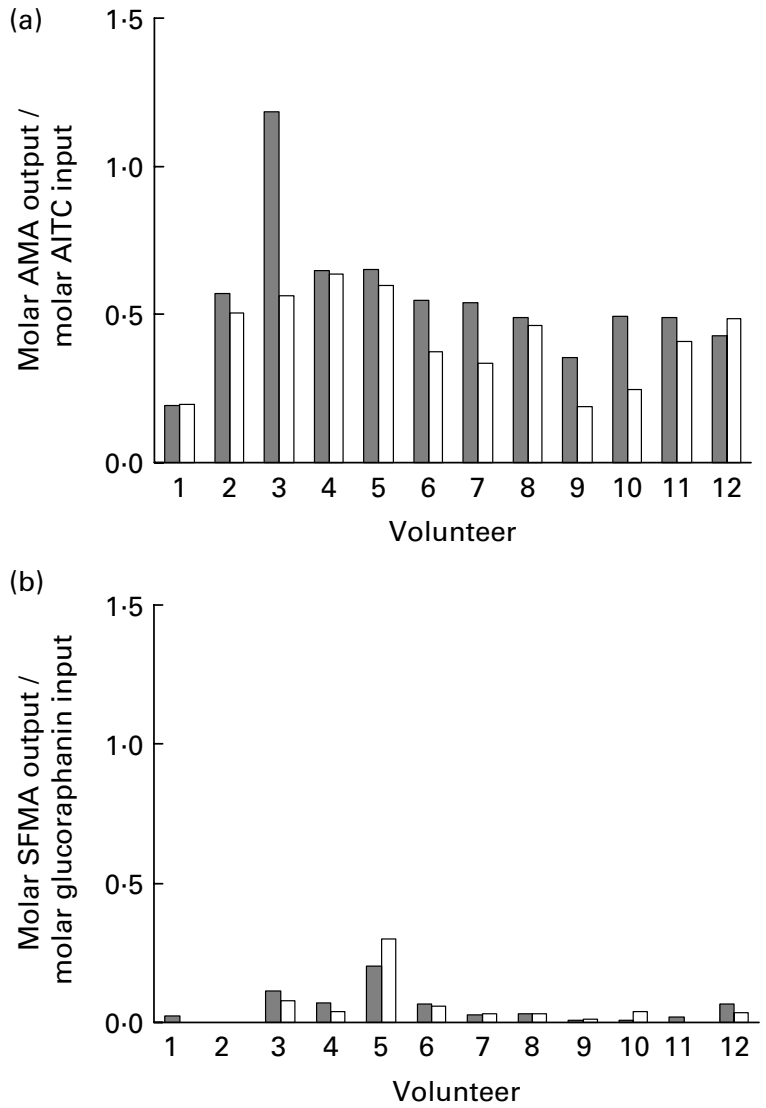

Fig. 5. Inter-individual variation $(P<0.001)$ in total excretion of (a) allyl mercapturic acid AMA ( $\mu \mathrm{mol}$ ) over $24 \mathrm{~h}$ as a proportion of allyl isothiocyanate (AITC) intake ( $\mu \mathrm{mol})$, and (b) sulforaphane mercapturic acid (SFMA; $\mu \mathrm{mol}$ ) over $24 \mathrm{~h}$ as a proportion of glucoraphanin intake ( $\mu \mathrm{mol})$, after consumption of fully cooked broccoli along with a meat-containing $(\square)$ or vegetarian $(\square)$ meal.

broccoli, the proportion of glucoraphanin excreted as SFMA in the first urine collection was similar to that in the second collection $(P=0 \cdot 372)$, but higher than in the fourth urine collection $(P<0.001)$. Following the consumption of fully cooked broccoli, it was higher in the second urine collection $(P<0.001)$, but was not different in the last urine collection $(P=0 \cdot 821)$. This trend caused a significant interaction between the effect of broccoli treatment and time of urine excretion on SFMA output $(P=0.002)$, irrespective of type of meal.

\section{Discussion}

Previous feeding trials with brassica, involving human subjects, have offered raw or cooked vegetables or both, and measured urinary excretion of MA of isothiocyanates (Getahun \& Chung, 1999; Conaway et al. 2000; Rouzaud et al. 2004). Brassica are usually consumed as part of a complex meal. An understanding of the influence of the meal matrix on the absorption of isothiocyanates is important to complement research on the metabolic fate of glucosinolates from processed brassica vegetables and cancer-prevention. In brassica, sulforaphane may be one of the most potent chemoprotective isothiocyanates (Zhang et al. 1992; Myzak \& Dashwood, 2006). 
The present study investigated the effect of cooking broccoli together with the influence of the meal matrix on the time-course of production of sulforaphane in healthy volunteers. The durations for cooking broccoli were based on results from a previous experiment in which the highest production of isothiocyanates following hydrolysis, in vitro, was in lightly cooked brassica compared with raw or fully cooked brassica (Rungapamestry et al. 2006). Production of sulforaphane was quantified using its urinary MA biomarker, SFMA, a specific means of measuring excretion of individual isothiocyanates compared with measurement of the output of total isothiocyanates by their cyclocondensation products, used in previous studies (Getahun \& Chung, 1999; Conaway et al. 2000; Shapiro et al. 2001). The current experiment is the first to show that the meal matrix can influence the production of pre-formed isothiocyanates in vivo.

Sulforaphane and AITC absorbed from cooked broccoli and mustard respectively were completely excreted $24 \mathrm{~h}$ after consumption of brassica by volunteers. This observation is in agreement with previous experiments on the time-course of excretion of MA after ingestion of glucosinolate precursors or isothiocyanates by human subjects (Chung et al. 1992; Conaway et al. 2000; Rouzaud et al. 2004). The peak in AMA excretion within $4 \mathrm{~h}$ of AITC intake and the proportion of ingested AITC converted to AMA after complete excretion of the metabolite are comparable to previous reports (Jiao et al. 1994).

Total AMA output, expressed as the molar proportion of AITC intake, was significantly influenced by the meal matrix but not by the cooking treatment of broccoli. The effect of meal composition on the postprandial absorption of isothiocyanates was expected to be partly dictated by the protein or fat content of the meal. Isothiocyanates have been shown to interact with proteins in vitro (Björkman, 1973). Contrary to our hypothesis that protein would delay or lower the absorption of isothiocyanates in the present experiment, excretion of AMA was higher after ingestion of AITC together with the meat-containing meal, which was higher in protein than the vegetarian meal. The fat content of the meat-containing meal was also 5-fold higher than that of the vegetarian meal. Isothiocyanates are relatively non-polar and the fat content of the meals may have been responsible for the subtle but significant differences in AITC uptake from the two types of meal. The improved bioavailability of many lipophilic drugs taken with food is often due to the lipid content of food, while lipid-based formulations can assist in the formation of solubilised phases for absorption of lipophilic drugs (Humberstone \& Charman, 1997). The higher lipophilicity of AITC, compared with sulforaphane, may partly explain the influence of meal composition on absorption of AITC but not on sulforaphane. Isothiocyanates may also be better incorporated into lipids during the chewing phase, if they are ingested preformed, rather than being formed during the later stages of digestion within the gastrointestinal chyme, after hydrolysis of their glucosinolate precursors.

Total SFMA output was approximately 3 and $10 \mu \mathrm{mol}$, corresponding to 5 and $20 \%$ of glucoraphanin intake, after consumption of fully cooked and lightly cooked broccoli respectively. Excretion of SFMA was therefore expected to be higher following the intake of the broccoli seed extract due to its expected high concentrations of pre-formed sulforaphane, as opposed to the comparably lower glucoraphanin concentrations in mature broccoli. However, trace concentrations of SFMA were observed due to the predominant yield of sulforaphane nitrile, which was 45 times higher than sulforaphane during preparation of the extract. The low concentration of sulforaphane in the seed extract was below the sensitivity of the MA analysis, compared with its yield from cooked broccoli.

Although mean intake of glucoraphanin was similar in lightly cooked or fully cooked broccoli, consumption of lightly cooked broccoli produced a higher yield of sulforaphane, expressed as the molar proportion of glucoraphanin ingested, in vivo. This observation is in agreement with previous experiments in human subjects, showing that production of isothiocyanates is higher following ingestion of brassica with active plant myrosinase, compared with those in which myrosinase has been denatured (Getahun \& Chung, 1999; Conaway et al. 2000; Shapiro et al. 2001; Rouzaud et al. 2004). In the current study, lightly cooked broccoli had a significantly higher plant myrosinase activity than fully cooked broccoli. No direct evidence for the release of isothiocyanates within the colon is available in vivo. Colonic microbiota has been estimated to account for about $8 \%$ of isothiocyanates recovered as MA in gnotobiotic rats harbouring a human faecal microbiota, compared with their germ-free counterparts, when given a diet with or without myrosinase (Rouzaud et al. 2003). This is comparable to the recovery of sulforaphane of $5 \%$ as SFMA after fully cooked broccoli in our experiment. It has been suggested that the lower recovery of isothiocyanates in the colon may be due to the predominant formation of nitriles (Cheng et al. 2004) or amines (Combourieu et al. 2001), as shown after glucosinolate hydrolysis by human feacal microbiota in vitro.

The output of AITC and sulforaphane, as a proportion of ingested precursors, was highly variable among volunteers. Inter-individual variability in SFMA output was more pronounced after consumption of fully cooked compared with lightly cooked broccoli, suggesting significant inter-individual variation in colonic hydrolysis of glucoraphanin. Large interindividual differences in faecal microbiota populations of genera such as Bacteroides, Bifidobacteria and Lactobacilli, implicated in glucosinolate hydrolysis (NugonBaudon et al. 1990; Elfoul et al. 2001; Cheng et al. 2004), have been reported among human subjects (Nagashima et al. 2003). Furthermore, isothiocyanate metabolism and excretion can vary markedly among individuals (Zhang et al. 1995). Inter-individual variability associated with the digestive fate of glucoraphanin was higher than that associated with the output of AITC. The AITC was delivered directly to the stomach and small intestine, where it was presumably more readily available for absorption from the uniform matrix of mustard. Conversely, sulforaphane was produced in the alimentary tract following hydrolysis of glucoraphanin in situ within the non-homogeneous matrix of cooked broccoli. The higher variability in SFMA compared with AMA excretion could therefore be a function of the combined effect of plant and microbial myrosinase in the former. It could be further explained by temporal differences in the degree of chewing and gastro-intestinal transit time among individuals. This larger inter-individual variation in SFMA may have masked any influence of meal composition on SFMA excretion. 
The output of AMA was considerably higher than that of SFMA, when expressed as a proportion of ingested precursors. Pre-formed AITC was readily available for absorption from the stomach and small intestine, while only a relatively small fraction of ingested glucoraphanin was hydrolysed to and absorbed as sulforaphane in vivo. Excretion of MA can be considerably higher after ingestion of pre-formed isothiocyanates than after consumption of their glucosinolate precursors (Shapiro et al. 2001). Furthermore, sulforaphane has been shown to have a slower metabolic disposal via its conjugation with glutathione at physiological $\mathrm{pH}$, compared with AITC (Zhang et al. 1995).

The findings from the present experiment suggest that the uptake of cancer-protective isothiocyanates following consumption of lightly cooked brassica vegetables is more extensive than after intake of the fully cooked vegetables. We have also shown that individuals vary extensively in the way in which they metabolise glucosinolates to isothiocyanates. Moreover, the nature of the meal matrix can considerably influence the digestive fate of pre-formed isothiocyanates. The main determinant in the production of isothiocyanates from their glucosinolate precursors was the way in which the brassica vegetable was cooked.

\section{Acknowledgements}

We thank the volunteers for their participation and compliance in this study. We acknowledge The Rowett Research Institute who provided the facilities for blast freezing the meals, Mrs Heather Scott (The Robert Gordon University) who helped in the preparation and serving of the meals, and Dr Jason Owen and Mrs Gillian Telfer (Macaulay Institute) for the analysis of nitrogen and fat content of the meals. We also thank Dr Mark Brewer (Biomathematics \& Statistics Scotland) for statistical advice. This study was supported by the Food Standards Agency (contract No. T01027, Influence of cooking and processing of brassica vegetables on the release of beneficial and harmful metabolites of glucosinolates in the digestive tract).

\section{References}

Björkman R (1973) Interaction between proteins and glucosinolate isothiocyanates and oxazolidinethiones from Brassica napus seed. Phytochem 12, 1585-1590.

British Standards (1996) Methods of test for meat and meat products. Determination of free fat content. BS4401-5. British Standards, London, UK.

Brüsewitz G, Cameron BD, Chasseaud LF, Görler K, Hawkins DR, Koch H \& Mennicke WH (1977) The metabolism of benzyl isothiocyanate and its cysteine conjugate. Biochem J 162, 99-107.

Cheng DL, Hashimoto K \& Uda Y (2004) In vitro digestion of sinigrin and glucotropaeolin by single strains of Bifidobacterium and identification of the digestive products. Food Chem Toxicol 42 , $351-357$

Chung FL, Morse MA, Eklind KI \& Lewis J (1992) Quantitation of human uptake of the anticarcinogen phenethyl isothiocyanate after a watercress meal. Cancer Epidemiol Biomarkers Prev 1, 383-388.

Combourieu B, Elfoul L, Delort AM \& Rabot S (2001) Identification of new derivatives of sinigrin and glucotropaeolin produced by the human digestive microflora using H-1 NMR spectroscopy analysis of in vitro incubations. Drug Metab Dispos 29, 1440-1445.

Conaway CC, Getahun SM, Liebes LL, Pusateri DJ, Topham DKW, Botero-Omary M \& Chung FL (2000) Disposition of glucosinolates and sulforaphane in humans after ingestion of steamed and fresh broccoli. Nutr Cancer 38, 168-178.

Duncan AJ, Rabot S \& NugonBaudon L (1997) Urinary mercapturic acids as markers for the determination of isothiocyanate release from glucosinolates in rats fed a cauliflower diet. $J$ Sci Food Agri 73, 214-220.

Elfoul L, Rabot S, Khelifa N, Quinsac A, Duguay A \& Rimbault A (2001) Formation of allyl isothiocyanate from sinigrin in the digestive tract of rats monoassociated with a human colonic strain of Bacteroides thetaiotaomicron. FEMS Microbiol Lett 197, 99-103.

European Union (1997) Oil seeds - determination of glucosinolates. Off J Eur Communities L170, 28-34.

Fenwick GR \& Heaney RK (1983) Glucosinolates and their breakdown products in cruciferous crops, foods and feedingstuffs. Food Chem 11, 249-271.

Genstat 5 Committee (2005) Genstat release 8.1. Rothamsted: Lawes Agricultural Trust.

Getahun SM \& Chung FL (1999) Conversion of glucosinolates to isothiocyanates in humans after ingestion of cooked watercress. Cancer Epidemiol Biomarkers Prev 8, 447-451.

Humberstone AJ \& Charman WN (1997) Lipid-based vehicles for the oral delivery of poorly water soluble drugs. Adv Drug Deliv Rev 25, $103-128$.

Jiao D, Ho CT, Foiles P \& Chung FL (1994) Identification and quantification of the N-Acetylcysteine conjugate of allyl isothiocyanate in human urine after ingestion of mustard. Cancer Epidemiol Biomarkers Prev 3, 487-492.

Krul C, Humblot C, Philippe C, Vermeulen M, van Nuenen M, Havenaar R \& Rabot S (2002) Metabolism of sinigrin (2-propenyl glucosinolate) by the human colonic microflora in a dynamic in vitro large-intestinal model. Carcinogenesis 23, 1009-1016.

MAFF (1988) Food Portion Sizes, 2nd ed. Norwich: HMSO.

Matusheski NV, Juvik JA \& Jeffery EH (2004) Heating decreases epithiospecifier protein activity and increases sulforaphane formation in broccoli. Phytochemistry 65, 1273-1281.

Mennicke WH, Gorler K \& Krumbiegel G (1983) Metabolism of some naturally-occurring isothiocyanates in the rat. Xenobiotica 13, 203-207.

Mennicke WH, Kral T, Krumbiegel G \& Rittmann N (1987) Determination of N-acetyl-S-(N-alkylthiocarbamoyl)-L-cysteine, a principal metabolite of alkyl isothiocyanates, in rat urine. J Chromatogr B Biomed Appl 414, 19-24.

Minchinton I, Sang J, Burke D \& Truscott RJW (1982) Separation of desulphoglucosinolates by reversed-phase high-performance liquid chromatography. J Chromatogr 247, 141-148.

Myzak MC \& Dashwood RH (2006) Chemoprotection by sulforaphane: Keep one eye beyond Keap1. Cancer Lett 233, 208-218.

Nagashima K, Hisada T, Sato M \& Mochizuki J (2003) Application of new primer-enzyme combinations to terminal restriction fragment length polymorphism profiling of bacterial Populations in human Feces. Applied and Environmental Microbiology 69, $1251-1262$.

NugonBaudon L, Rabot S, Wal JM \& Szylit O (1990) Interactions of the intestinal microflora with glucosinolates in rapeseed meal toxicity - 1st evidence of an intestinal Lactobacillus possessing a myrosinase-like activity in vivo. J Sci Food Agri 52, 547-559.

Pella E \& Colombo B (1973) Study of carbon, hydrogen and nitrogen by combustion gas-chromatography. Mikrochim Acta 697-719.

Rouzaud G (2001) Digestive metabolism of glucosinolates: A novel approach using urinary markers for estimating the release of glucosinolate breakdown products in the gastrointestinal tract of mammals. PhD Thesis, The Robert Gordon University, UK. 
Rouzaud G, Rabot S, Ratcliffe B \& Duncan AJ (2003) Influence of plant and bacterial myrosinase activity on the metabolic fate of glucosinolates in gnotobiotic rats. Br J Nutr 90, 395-404.

Rouzaud G, Young SA \& Duncan AJ (2004) Hydrolysis of glucosinolates to isothiocyanates after ingestion of raw or microwaved cabbage by human volunteers. Cancer Epidemiol Biomarkers Prev 13, $125-131$.

Rungapamestry V, Duncan AJ, Fuller Z \& Ratcliffe B (2006) Changes in glucosinolate concentrations, myrosinase activity and production of metabolites of glucosinolates in cabbage (Brassica oleracea var. capitata) cooked for different durations. J Agric Food Chem 54, 7628-7634.

Shapiro TA, Fahey JW, Wade KL, Stephenson KK \& Talalay P (2001) Chemoprotective glucosinolates and isothiocyanates of broccoli sprouts: Metabolism and excretion in humans. Cancer Epidemiol Biomarkers Prev 10, 501-508.

van Poppel G, Verhoeven DTH, Verhagen H \& Goldbohm RA (1999) Brassica vegetables and cancer prevention - Epidemiology and mechanisms. Advances in Nutrition and Cancer 2 472, 159-168.

Zhang YS, Kolm RH, Mannervik B \& Talalay P (1995) Reversible conjugation of isothiocyanates with glutathione catalyzed by human glutathione transferases. Biochem Biophys Res Commun 206, 748-755.

Zhang YS, Talalay P, Cho CG \& Posner GH (1992) A major inducer of anticarcinogenic protective enzymes from broccoli - isolation and elucidation of structure. Proc Natl Acad Sci U S A 89, 2399-2403.

Zhang YS, Yao S \& Li J (2006) Vegetable-derived isothiocyanates: anti-proliferative activity and mechanism of action. Proc Nutr Soc 65, 68-75. 